Research Paper

\title{
Overexpression of PTK6 predicts poor prognosis in bladder cancer patients
}

\author{
Xue-lian $\mathrm{Xu}^{1 *}$, Yun-Lin $\mathrm{Ye}^{2 *}$, Zhi-Ming Wu ${ }^{2}$, Qiu-Ming He², Lei Tan², Kang-Hua Xiao' ${ }^{2}$, Rui-yan Wu ${ }^{1}$, Yan \\ $\mathrm{Yu}^{1}$, Jia Mai ${ }^{1}$, Zhi-ling Li ${ }^{1}$, Xiao-dan Peng ${ }^{1}$, Yun Huang ${ }^{1}$, Xuan Li ${ }^{1}$, Hai-liang Zhang ${ }^{1}$, Xiao-feng Zhu ${ }^{1 凶}$, \\ Zi-Ke Qin ${ }^{2 \bowtie}$ \\ 1. State Key Laboratory of Oncology in South China, Collaborative Innovation Center for Cancer Medicine, Cancer Center, Sun Yat-sen University, \\ Guangzhou, 510060, China \\ 2. Department of Urological Oncology, Sun Yat-sen University Cancer Center, Guangzhou, 510060, China \\ *These authors have contributed equally to this work
}

$\triangle$ Corresponding authors: Xiao-Feng Zhu, M.D. \& Ph.D, State Key Laboratory of Oncology in South China, Sun Yat-sen University Cancer Center, 651 Dongfeng road east, Guangzhou 510060, Guangdong Province, China. Email: zhuxf@sysucc.org.cn and Zi-Ke Qin, MD \& Ph.D, Department of Urological Oncology, Sun Yat-sen University Cancer Center, 651 Dongfeng road east, Guangzhou 510060, Guangdong Province, China. Email: qinzk@sysucc.org.cn

(c) Ivyspring International Publisher. This is an open access article distributed under the terms of the Creative Commons Attribution (CC BY-NC) license (https://creativecommons.org/licenses/by-nc/4.0/). See http://ivyspring.com/terms for full terms and conditions.

Received: 2017.06.04; Accepted: 2017.07.22; Published: 2017.09.27

\begin{abstract}
Protein tyrosine kinase 6 (PTK6) is a non-receptor tyrosine kinase and works as an oncogene in various cancers. Recently, PTK6 has been used as a therapeutic target for breast cancer patients in a clinical study. However, the prognostic value of PTK6 in bladder cancer (BC) remains vague. Therefore, we retrieved 3 independent investigations of Oncomine database and found that PTK6 is highly expressed in BC tissues compared with corresponding normal controls. Similar results were also observed in clinical specimens at both mRNA and protein levels. Immunohistochemical analysis indicated that PTK6 overexpression was highly related to the T classification, $N$ classification, grade, recurrence, and poor prognosis of BC patients. Furthermore, we demonstrated that when PTK6 expression was knocked down by siRNAs, cell proliferation and migration were considerably inhibited in BC cell lines T24 and EJ. By these approaches, we are intended to elucidate PTK6 may be a reliable therapeutic target in BC and might benefit from PTK6 inhibitors in the future.
\end{abstract}

Key words: Bladder cancer, PTK6, proliferation, prognosis, Oncomine, GEO

\section{Introduction}

Bladder cancer (BC) is one of the most fatal malignant tumors and a serious public health problem causing an increasing incidence and mortality worldwide [1-3]. Environmental or occupational exposures to carcinogens, especially tobacco, are the main risk factors for bladder cancer [4]. In spite of advances in diagnostic and treatment strategies, surgical operation is still the most effective treatment therapy for $\mathrm{BC}[5,6]$, and only a handful of chemotherapy drugs are useful for $\mathrm{BC}$ patients which always result in an unsatisfactory clinical outcomes [7-10]. Consequently, explore effective prognostic biomarkers which can be utilized to refractory BC is needed.
Protein tyrosine kinase 6 (PTK6) is a non-receptor tyrosine kinase [11] and has both kinase-dependent and kinase-independent functions [12] which was first cloned from a metastatic human breast tumor $[13,14]$. Previous studies showed that PTK6 plays an important role of oncogene [15-18] or anti-oncogene [19-21] in numerous tumors. These studies demonstrated the involvement of PTK6 in cancer proliferation, metastasis and apoptosis [15, 22]. However, the prognostic significance of PTK6 in bladder cancer is still unclear.

In this study, we evaluated the expression of PTK6 at both the mRNA and protein levels. We further evaluated the relationship between PTK6 
expression and clinicopathologic parameters including prognostic significance, and explored the biological function of PTK6 in BC patients.

\section{Materials and Methods}

\section{Databases}

The datasets in Oncomine Cancer Microarray Database (https://www.oncomine.org) were used to identify the expression pattern of PTK6 in BC. The datasets of corresponding clinical data used throughout this study was downloaded from the GEO database (https://www.ncbi.nlm.nih.gov/geo/) following approval of these projects by the consortium. To gain further insight into the biological pathways involved in BC pathogenesis through PTK6 pathway, a gene set enrichment analysis (GSEA) was performed. The canonical pathways gene sets (h.all.v6.0.symbols.gmt) from the Molecular Signatures Database-MsigDB (http://software.broad institute.org/gsea/msigdb/collections.jsp\#H) were used for enrichment analysis.

\section{RNA extraction, reverse transcription and real time PCR}

Total RNAs from fresh tissues were purified from tissues using TRIzol Reagent (Invitrogen, Carlsbad, CA, USA) according to the manufacturer's instructions, and $2 \mu \mathrm{g}$ RNA of each sample was reverse transcribed using SuperScript RT kit (Invitrogen Life Technologies, Carlsbad, CA, USA). Full-length open reading frame of PTK6 was amplified by PCR from cDNA samples of normal bladder epithelium tissues and bladder cancer tissues. Real-time PCR was performed using a CFX96 Real-Time System (BIO-RAD) (PREMIER Biosoft International, Palo Alto CA, USA). Sequences of the primers were as follows: PTK6, 5'-TACTTTGGGGAGGTCTTCGAG-3' (sense), 5'-TBCCBCABCTTCTTCATG-3'(antisense); GAPDH, 5'-GACTCATGACCACAGTCCATBC-3' (sense), 5'-AGAGBCAGGGATGATGTTCTG-3' (antisense). We utilized the SYBR Green kit (Invitrogen Life Technologies, Carlsbad, CA, USA) to execute the amplification of the cDNA. The RT-PCR cycling parameters were performed as follows: denaturation at $95^{\circ} \mathrm{C}$ for 15 seconds, annealing at $55^{\circ} \mathrm{C}$ for 30 seconds and extension at $72^{\circ} \mathrm{C}$ for 30 seconds. The expression data were normalized to the geometric mean of housekeeping gene GAPDH to control the difference in expression levels and analyzed using the 2-Delta Delta $\mathrm{C}(\mathrm{T})$ method.

\section{Tumor specimens and patient data}

151 paraffin-embedded archived samples of BC were used in immunohistochemistry, including 15 samples with adjacent normal tissues. All these samples were derived from initial surgery without either preoperative chemotherapy or radiotherapy between 2000 and 2010 at SYSUCC. TNM staging and clinicopathologic classification were determined according to the 2010 American Joint Committee on Cancer (AJCC) staging system. Clinicopathologic information of the $151 \mathrm{BC}$ samples was described in Table

Overall survival (OS) was defined as the interval between the date of surgery and the date of death or the last known follow up. Additionally, 15 pairs of matched tumor samples and adjacent normal tissues (collected immediately after surgery and stored at $-80^{\circ} \mathrm{C}$ were used for real time PCR analysis.

151 patients included 135 males and 16 females from 15 to 87 years (mean, 61 years). Of these patients, 79 patients underwent radical cystectomy, 22 patients underwent partial cystectomy, and 50 patients underwent TURBT (transurethral resection of bladder tumor). After partial cystectomy and TURBT, mitomycin $\mathrm{C}$ was used in intravesical therapy as weekly intravesical injection beginning within 24 hours after surgery. Thirty specimens of adjacent normal bladder tissue distant from the tumor were included for these patients, as well. The median follow-up time for overall survival was 66.5 months for patients still alive at the time of analysis, range from 1 to 182 months.

\section{Cell culture and transfection}

T24, BIU87 and EJ were purchased from the Cell Bank of Type Culture Collection of Chinese Academy of Sciences, Shanghai, China, which were grown in 1640 (Invitrogen) supplemented with 5\% fetal bovine serum in a damp incubator containing 5\% CO2 at $37^{\circ} \mathrm{C}$. The Normal cell is a primary cell which is purified from the no bladder cancer patient. The sequences of oligonucleotides1 and 2 targeting PTK6 mRNA, are Si\#1: GGUGCUAUCACAUCUAUAU; Si\#2: GGACAGTGCTCACGACACAACCAAA; These siRNAs were synthesized by GenePharma. Approximately $2 \times 10^{5}$ cells per well were seeded in a 6-well tissue culture dish on the day before transfection. Transfection of 50nmol siRNAs was performed according to the manufacturer's instructions by using the LipofectamineTM transfection reagent (Invitrogen).

\section{Western blotting and antibodies}

Western blotting was performed as described previously Western blotting analysis was carried out with proteins collected from the adjacent normal epithelium tissues and the cancer tissues, and total proteins were Tris- $\mathrm{HCl}(\mathrm{pH} / 6.8), 5 \%$ 2-mercapto- 
ethanol, and $10 \%$ glycerol. The concentration of the protein was measured by the BCA protein assay kit (PIERCE, Rockford, IL, USA). A total of $20 \mu \mathrm{g}$ protein was electrophoretically separated in $12 \%$ SDS polyextracted with $1 \mathrm{X}$ sodium dodecyl sulfate (SDS) sample buffer ( $2 \%$ SDS, $62.5 \mathrm{mmol} / \mathrm{l})$ acrylamide gels and transferred onto polyvinylidene difluoride membranes (Amersham Pharmacia Biotech, Piscataway, NJ). Then incubate the primary polyclonal antibody against PTK6 (dilution, 1:1000; ABGENT INC, USA), which was mixed with PTK6 Antibody (N-term) Blocking Peptide $(0.25 \mathrm{ug} / \mathrm{ml})$, in $5 \%$ of the skimmed milk solution overnight at $4^{\circ} \mathrm{C}$, and anti-rabbit (1:3000; Santa Cruz Biotechnology, Santa Cruz, CA) secondary antibody was used to detect PTK6 protein. Anti-glyceraldehyde 3-phosphate dehydrogenase (GAPDH) (1:3000, Santa Cruz, CA, USA) antibody and anti-mouse (1:3000; Santa Cruz Biotechnology, Santa Cruz, CA) secondary antibody were utilized to confirm equal loading. The protein signals were observed by the enhanced chemiluminescence (ECL) detection system (Amersham Biosciences Europe, Freiburg, Germany) according to the manufacturer's protocols.

\section{Cell proliferation, wound-healing and migration assays}

The viability of cells was evaluated by Cell Counting Kit-8 (CCK8, Dojindo Molecular Technologies, Japan) according to the manufacturer's instructions. Tumor cells were seeded onto 96-well plates at a density of $4 \times 103$ cells per well. CCK8 solution was added to each well at the time points of $24 \mathrm{~h}, 48 \mathrm{~h}, 72 \mathrm{~h}$, and $96 \mathrm{~h}$. The plate was examined at $450 \mathrm{~nm}$ absorption with a microplate reader after incubating for 4 hours at $37^{\circ} \mathrm{C}$.

Cells were trypsinized and seeded equally into 6-well plates to grow to almost full confluence in 24 hours, followed by non-serum starvation for another 24 hours. The cell monolayer was subsequently scratched with a sterile $100 \mathrm{ml}$ pipette tip. After scratching, the cells were washed with PBS and then cultured in a serum-free medium. Cell migration images were captured at time points of $0 \mathrm{~h}$ and $48 \mathrm{~h}$ by an inverted microscope (100X). For the transwell migration assay, $1.5 \times 10^{5}$ cells in $200 \mu$ l of serum-free DMEM were added to the cell culture inserts with an $8-\mu \mathrm{m}$ microporous filter without extracellular matrix coating (Becton Dickinson Labware, Bedford, MA). The DMEM medium containing $10 \%$ FBS was added to the bottom chamber. After 36 hours of incubation, the cells on the lower surface of the filter were fixed and stained prior to microscopic examination. The number of migrated cells in three random optical fields $(100 \mathrm{X})$ for each filter from triplicate filters was averaged.

\section{Immunohistochemistry (IHC) and scoring}

Immunohistochemistry was performed to examine the PTK6 expression in 150 bladder cancer tissue specimens. PTK6 was detected using a rabbit monoclonal antibody against PTK6 (ABGENT INC, AP7715a USA). Briefly, paraffin-embedded specimens were cut into $4 \mu \mathrm{m}$ sections and baked at $65^{\circ} \mathrm{C}$ for $30 /$ minutes, and a paraffin section of the bladder cancer tissue from patients was de-paraffined with xylene and rehydrated through graded alcohol. Then put in the blocking buffer ( $3 \%$ hydrogen peroxide) for endogenous peroxidase in $15 /$ minutes. Use the high-temperature heat technique with citric acid to antigenic retrieval, then cooling naturally. The tissue slides were incubated with anti-PTK6 antibody at a dilution of $1: 100$ for 12 /hours at $4^{\circ} \mathrm{C}$ in a moist chamber $(\mathrm{O} / \mathrm{N})$, and the anti-PTK6 antibody was mixed with PTK6 Antibody (N-term) Blocking Peptide $(2.5 \mathrm{ug} / \mathrm{ml})$. Subsequently, biotinylated anti-rabbit secondary antibody was applied for $35 /$ minutes at $37^{\circ} \mathrm{C}$. Then, the sections were incubated with streptavidin-horseradish peroxidase complex and developed with 3-diaminobenzidinetetrahydrochloride (DAB) for /minutes. Mayer's hematoxylin was applied as a counterstain utilizing Olympus CX31 microscope (Olympus, Center Valley, PA). Cytoplasmic PTK6 was evaluated according to the percentage of stained cells (median, 60\%; range, 0 to $100 \%$ ) and staining intensity: negative staining; low staining, light yellow; intermediate staining, yellow brown; and high staining, brown. The proportion of immune-positive cells (categorized as follows: 0 , none; $1, \geq 1 \%$ to $<25 \%$; $2, \geq 25 \%$ to $<50 \% ; 3, \geq 50 \%$ to $<75 \%$; and $4, \geq 75 \%$ ) and The intensity of staining were scored on a scale of 1 (no staining), 2 (weak staining), 3 (moderate staining), 4 (strong staining). According to biological behavior of bladder cancer, all bladder cancer patients were divided into two groups (1-8.5 low expressing; and $>8.5$, high PTK6 expressing). Each section was evaluated by two independent pathologists without knowledge of the clinical features of the cases.

\section{Follow-up and clinical outcomes assessment}

The overall survival time (OS) was defined as the time between surgery and the last follow-up time or date of death. In our 150 patients, 72 cases performed the bladder-sparing operation. The Cystoscopy was performed every 3 months for the first 2 years, every 6 months for 2-5 years, and then annually in the 72 patients after the treatment. Another 79 patients, who were performed with Radical cystectomy, do the CT of pelvis every 3 months for the first 2 years, every 6 
months for 2-5 years, and then annually. The recurrence was defined by histopathologic findings the abnormal bladder mucosa or tissue of the tumor post-operatively.

\section{Statistical analysis}

All the statistical analyses were performed using the SPSS 17.0 software (SPSS Inc, Chicago, IL, USA) and GraphPad Prism 5 (San Diego, CA) software. The paired T-test was used to analyze the significance of PTK6 on protein and mRNA levels in the paired samples. The chi-square test was utilized to analyze the relationship between the PTK6 expression and clinic-pathological factors. Kaplan-Meier and log-rank tests were used for survival analysis. Multivariate Cox regression analysis was performed for all variables that were found to be significant by Univariate analysis. $\mathrm{P}$ values less than 0.05 were considered statistically significant.

\section{Results \\ PTK6 expression analysis in Oncomine database and The Cancer Cell Line Encyclopedia (CCLE) project}

The mRNA levels of PTK6 in Oncomine database were analyzed. In bladder cancer, PTK6 level was found higher in $\mathrm{BC}$ compared with adjacent normal bladder tissues in 3 bladder cancer datasets. Within the datasets, mRNA expression levels of PTK6 were up-regulated in the superficial or infiltrating tumor tissues compared with normal bladder (Figure 1A-D). Detail statements for 3 bladder cancer datasets information have been listed in the Table 1 . Additionally, CCLE analysis was in agreement with that of Oncomine indicating that PTK6 was up-regulated in bladder cancer cell lines (Figure 1E).

A

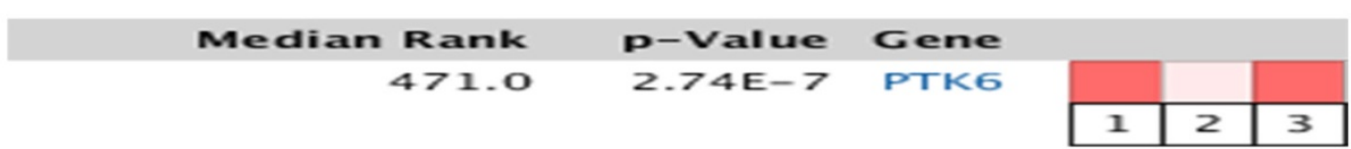

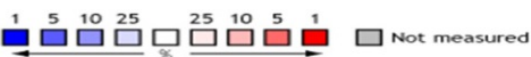

B

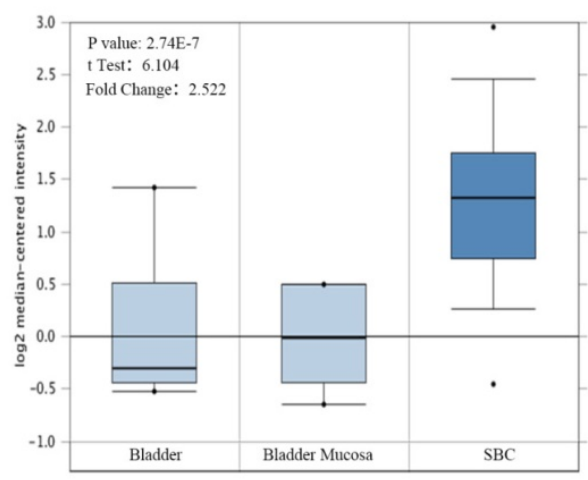

D

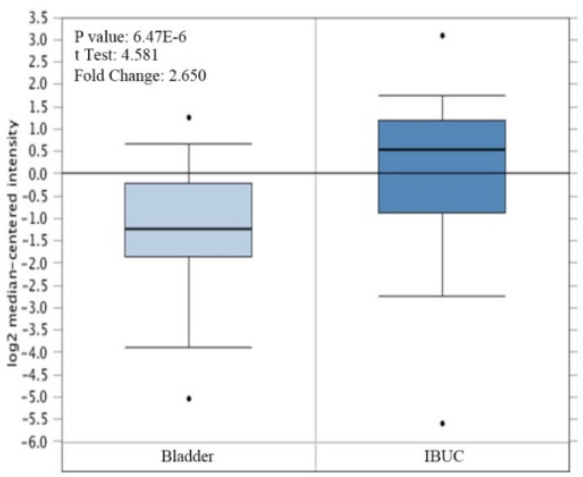

C

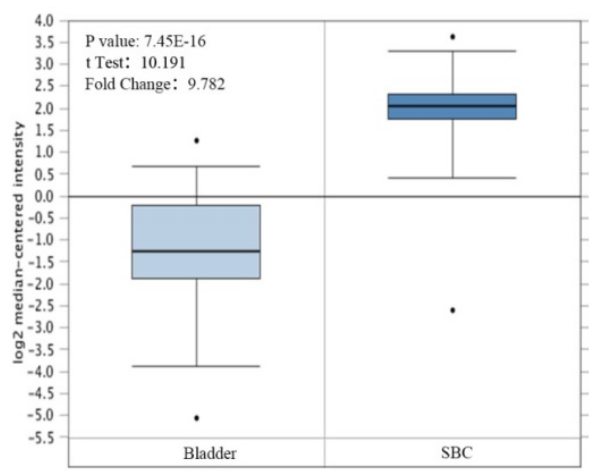

E

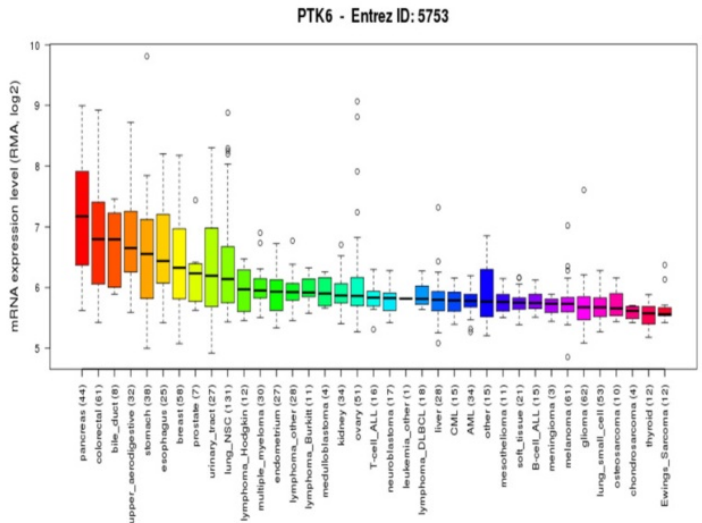

Figure 1. The expression of PTK6 in BC (Oncomine database). (A) A meta-analysis of PTK6 gene expression from six Oncomine databases where colored squares indicate the median rank for PTK6 (vs. Normal tissue) across 3 analyses. Sanchez-Carbayo Bladder 2 (1, 2), Dyrskjot Bladder 3 (3). The P value is given for the median rank analysis. (B) Comparison of PTK6 mRNA expression in Dyrskjot Bladder 3. (C, D) Comparison of PTK6 mRNA expression in Sanchez-Carbayo Bladder 2. (E) The mRNA expression level of PTK6 in a variety of cancer cell line (shown in arrow). Superficial bladder cancer (SBC); Infiltrating Bladder Urothelial Carcinoma (IBUC). 
Table 1. Detailed information about the 6 public expression datasets of Oncomine database about PTK6 in BC

\begin{tabular}{|c|c|c|c|c|}
\hline $\begin{array}{l}\text { Datasets (sample } \\
\text { size) }\end{array}$ & Comparison groups & $\begin{array}{l}\text { Fold } \\
\text { change }\end{array}$ & P value & $\begin{array}{l}\text { Overexpression } \\
\text { gene rank }\end{array}$ \\
\hline $\begin{array}{l}\text { Dyrskjot Bladder } \\
3(60)\end{array}$ & $\begin{array}{l}\text { Superficial Bladder } \\
\text { Cancer vs. Normal }\end{array}$ & 2.522 & $2.74 \mathrm{E}-7$ & 471 (in top $4 \%$ ) \\
\hline $\begin{array}{l}\text { Sanchez-Carbayo } \\
\text { Bladder 2(157) }\end{array}$ & $\begin{array}{l}\text { Infiltrating Bladder } \\
\text { Urothelial Carcinoma } \\
\text { vs. Normal }\end{array}$ & 2.650 & $6.47 \mathrm{E}-6$ & $\begin{array}{l}1332 \text { (in top } \\
11 \%)\end{array}$ \\
\hline $\begin{array}{l}\text { Sanchez-Carbayo } \\
\text { Bladder 2(157) }\end{array}$ & $\begin{array}{l}\text { Superficial Bladder } \\
\text { Cancer vs. Normal }\end{array}$ & 9.782 & 7.45E-16 & 429 (in top $4 \%$ ) \\
\hline
\end{tabular}

\section{Up-regulated expression of PTK6 in bladder cancer tissues}

To investigate the role of PTK6 in tumorigenesis, the expression of PTK6 in both mRNA and protein levels was evaluated in 15 pairs of $\mathrm{BC}$ tissues and the paracancerous epithelium tissues by real time PCR and western blotting. It is obvious that the expression of PTK6 was higher in BC than that in paracancerous epithelium tissues (Figure 2A-B).

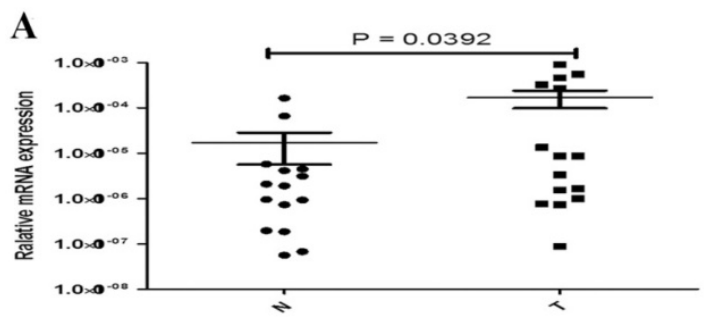

C

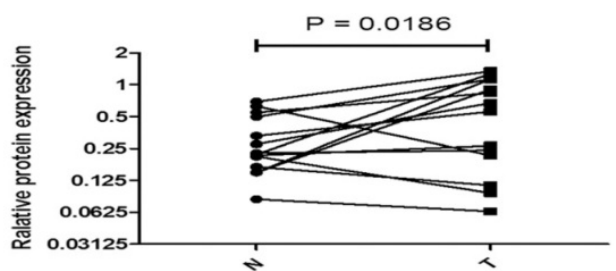

D

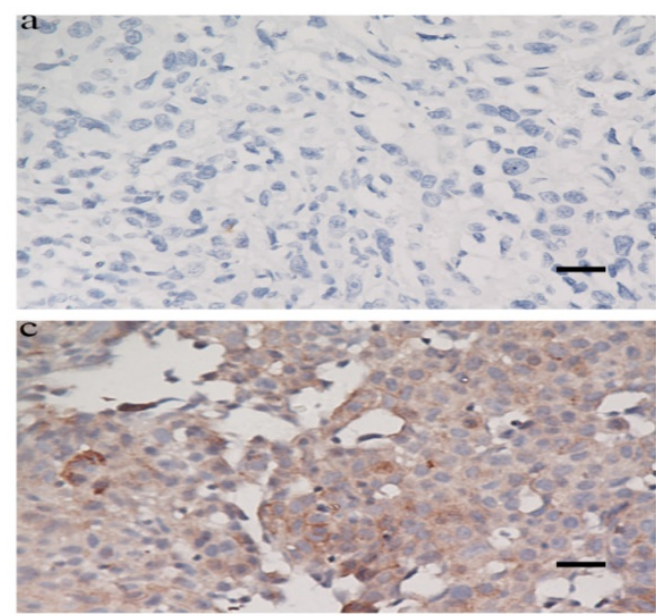

We further examined the expression of PTK6 protein in 151 paraffin-embedded bladder cancer samples and 5 matched paracancerous epithelial samples by immunohistochemical analysis. PTK6 expression was substantially up-regulated in the tumor tissues compared with the paracancerous epithelium tissues (Figure 2C, $\mathrm{p}<0.001$ ).

\section{Correlation between PTK6 expression and clinicopathologic features in tissue samples}

Clinical characteristics of BC included 70 cases of non-muscle invasive $(46.4 \%)$ and 81 cases of muscleinvasive $(53.6 \%)$ BC. 56 of total 151 BC cases $(37.1 \%)$ highly expressed PTK6; 95 cases $(62.9 \%)$ had low expression of PTK6 (Table 2). A strong correlation occurred between PTK6 expression (determined by using IHC staining), and clinicopathologic characteristics of these $\mathrm{BC}$ patients, including $\mathrm{T}$ classification $(p=0.002), N$ classification $(p=0.007)$, recurrence $(\mathrm{P}=0.039)$, and mortality $(\mathrm{P}<0.001)$ (Table 2). In contrast, PTK6 expression did not correlate with gender, age, grade and surgery type (Table 2).
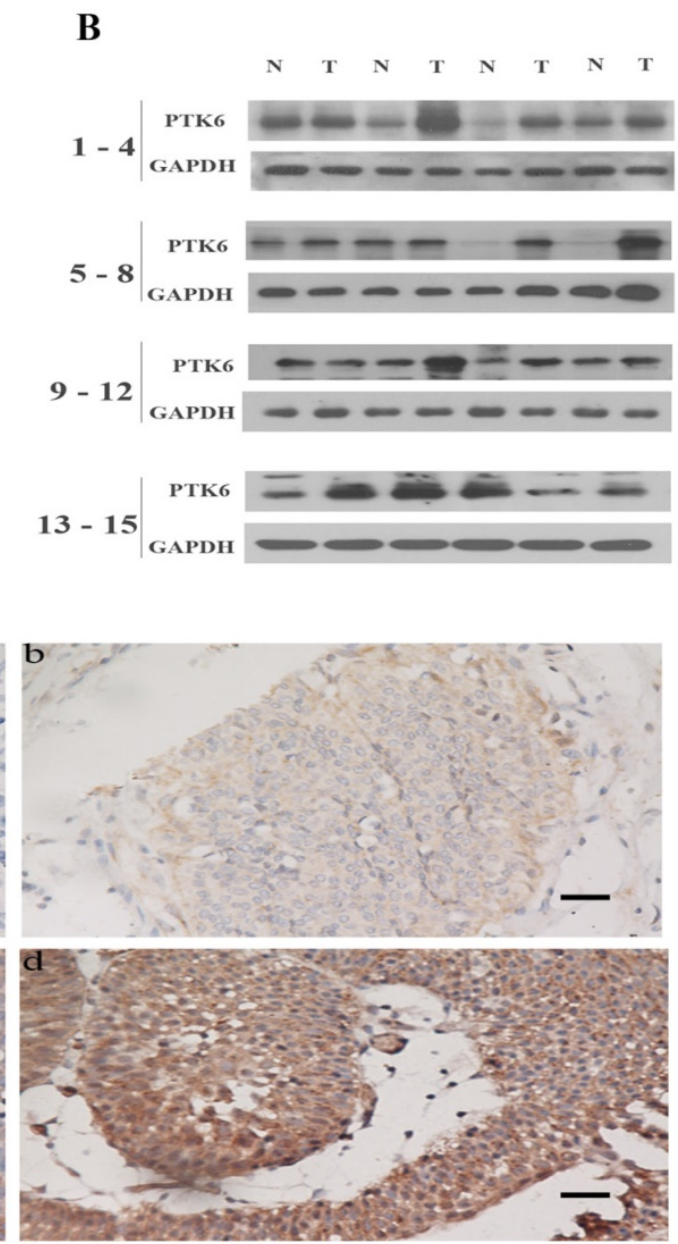

Figure 2. PTK6 is up-regulated in BC tissues. (A) Upregulated PTK6 mRNA expression in 15 matched tumor and paracancerous epithelium tissues. (B) Western blots show significantly upregulated protein expression of PTK6 in 15 pairs of resection specimens from BC patients. (C) The relative quantity of PTK6 protein was determined by densitometric analysis. (D) Immunohistochemical representative images of PTK6 expression in BC samples (a) BC, scored as (-); (b) BC, scored as (+); (c) BC, scored as (++); (d) BC, scored as (+++) (scale bar, $100 \mu \mathrm{m})$. 


\section{Association between PTK6 expression and patient survival}

A clear positive correlation existed between PTK6 expression and the overall survival of patients. Higher PTK6 expression related to a shorter overall survival time (OS) (median OS: 74 months) compared with lower PTK6 expression (median OS: 178 months) (Figure 3A, $\mathrm{p}<0.001$ ). Additionally, we determined the correlation between PTK6 expression and overall survival in BC patients regardless of age, histological differentiation, stage and recurrence. Kaplan-Meier analyses showed that overall survival is shorter in BC patients with higher PTK6 expression independent of histological differentiation and recurrence (Figure 3B-E). Univariate Cox regression analyses revealed that PTK6 expression, age, $\mathrm{T}$ classification, N classification, Grade, Surgery type and recurrence were significantly associated with overall survival (Table 3). Moreover, a multivariate Cox regression analysis confirmed PTK6 expression, grade and recurrence as independent predictors of overall survival in patients with BC (Table 3 ).

\section{PTK6 knockdown inhibits proliferation and cell migration in vitro}

To gain insight into the biological pathway

A
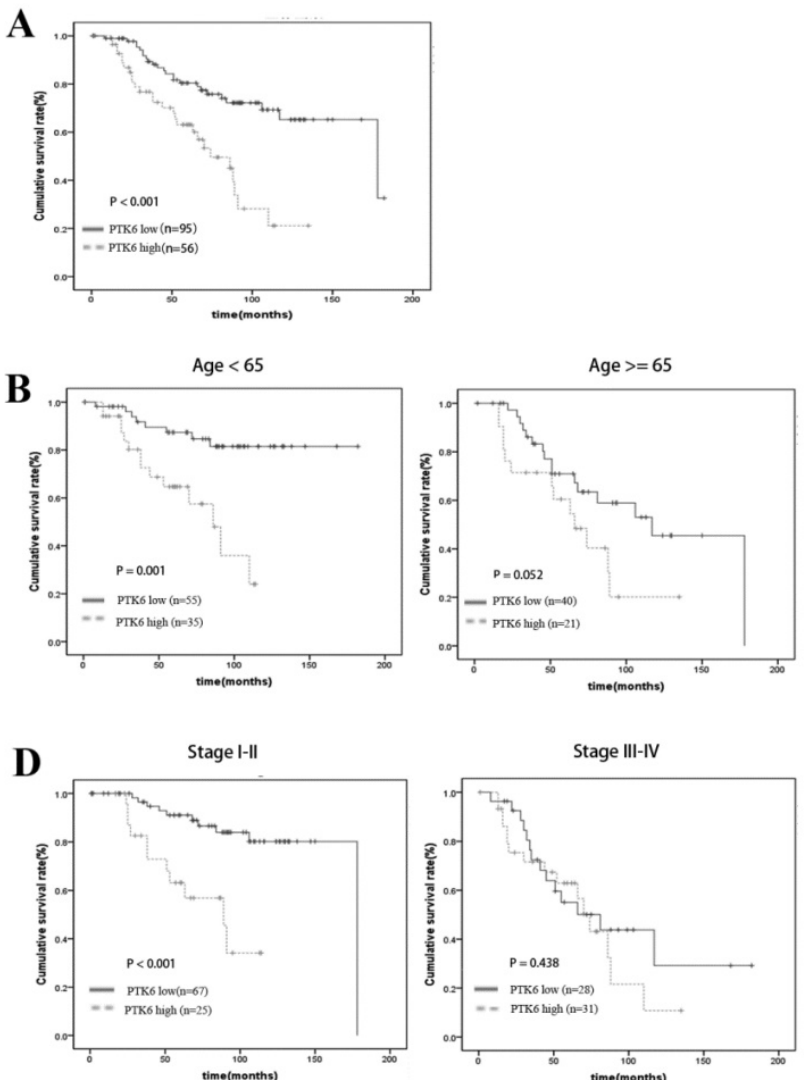

involved in $\mathrm{BC}$ pathogenesis stratified by the median of PTK6 expression level, GSEA analysis was conducted in GSE3167. Enrichment plots of GSEA showed that the gene signatures of cell proliferation, energy metabolism and apoptosis signaling pathway (Figure 4). To validate the GSEA analysis of PTK6, we assessed the tumorigenic ability of PTK6 in BC cells by CCK-8 proliferation assays. Firstly, we determined PTK6 expression in four BC cell lines at the protein level. Compared with Biu87, the expression of PTK6 was higher in T24 and EJ cells (Figure 5A). Then, we transfected T24 and EJ cells with PTK6 siRNAs, and western blot analysis verified PTK6 protein was markedly down-regulated in both T24 and EJ cells transfected with the two siRNAs (Figure 5B). Figure 5C shows that the proliferation of T24 and EJ cells transfected with siRNA\#1 or \#2 was significantly inhibited compared with that of the control group, indicating that PTK6 contributed greatly to the proliferation of the BC cells. Additionally, transwell migration and wound-healing assays showed that the migration rate of control cells was greater than that of PTK6-depleted BC cells by two siRNAs. These results indicate that oncogene PTK6 is required for cell growth and migration in $\mathrm{BC}$ cells.
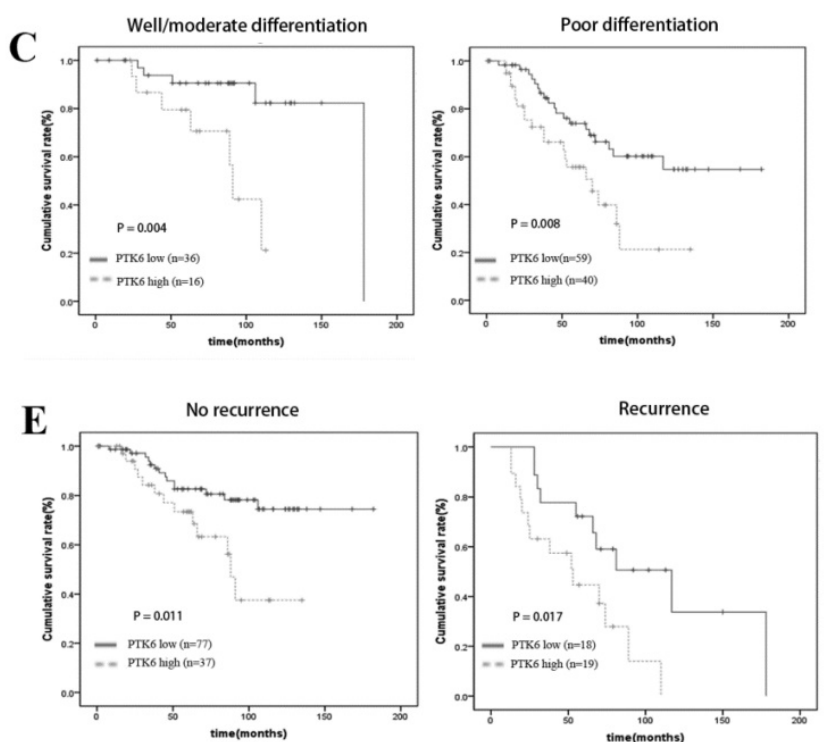

Figure 3. Kaplan-Meier analysis of overall survival in BC patients. (A) Overall survival analysis of 151 BC patients with different PTK6 protein expression. (Log-rank test: $\mathrm{P}<0.0001$ ). (B-E) Comparisons of overall survival between PTK6 high expression and PTK6 low expression in BC patients regardless of age, histological differentiation, stage and recurrence. 
A

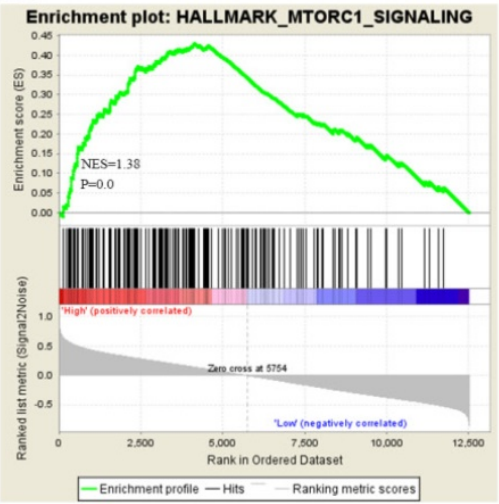

D

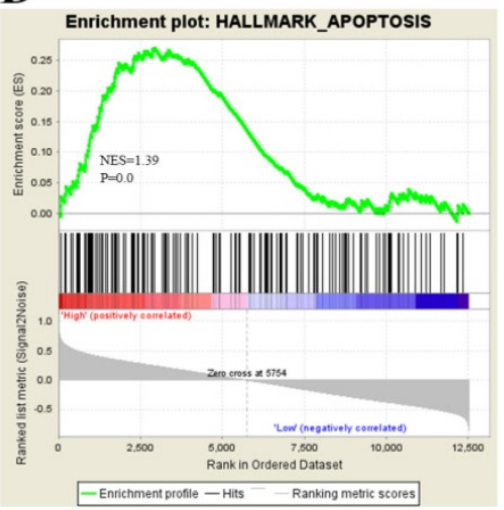

B

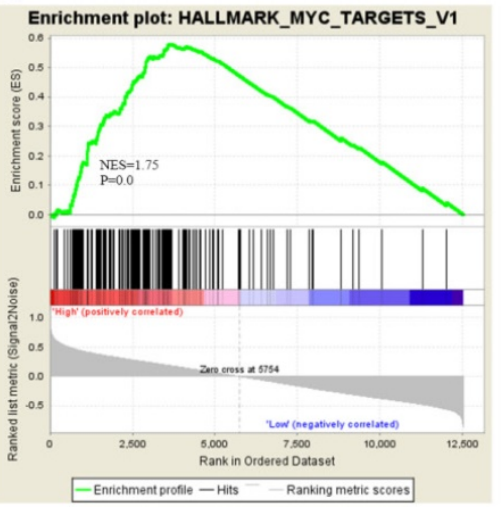

E

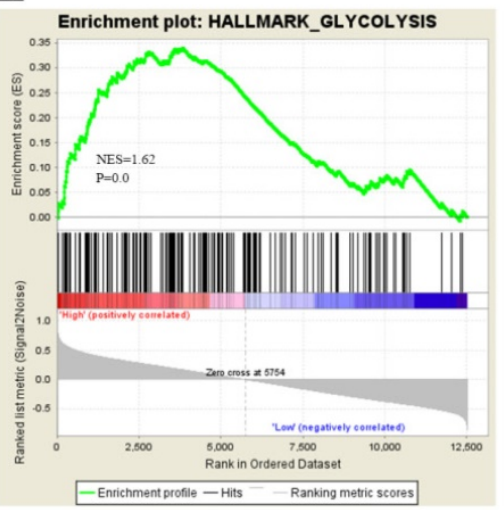

C

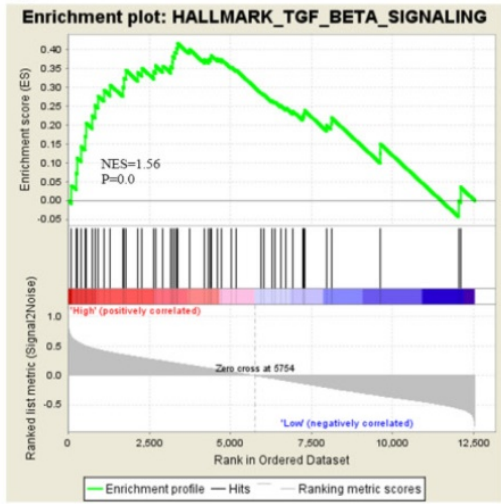

F

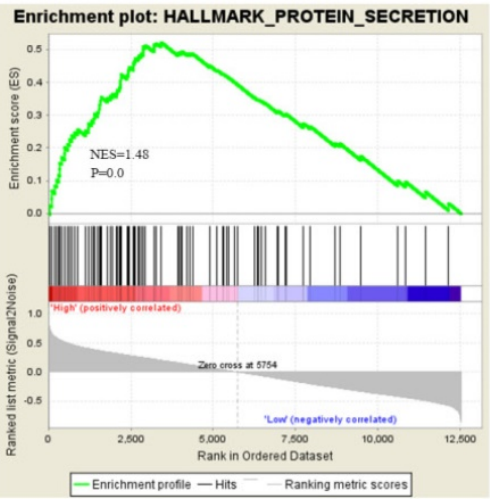

Figure 4: Biological functions of PTK6 in BC. (A-F) GSEA analysis on GSE3167 dataset showed the association of PTK6 expression with activity of the cell proliferation, energy metabolism and apoptosis signaling pathway. The normalized enrichment score (NES) means the degree to which the gene set is overrepresented at the top or bottom of the ranked list of genes.

Table 2. Association of PTK6 expression with the clinicopathological characteristics of BC

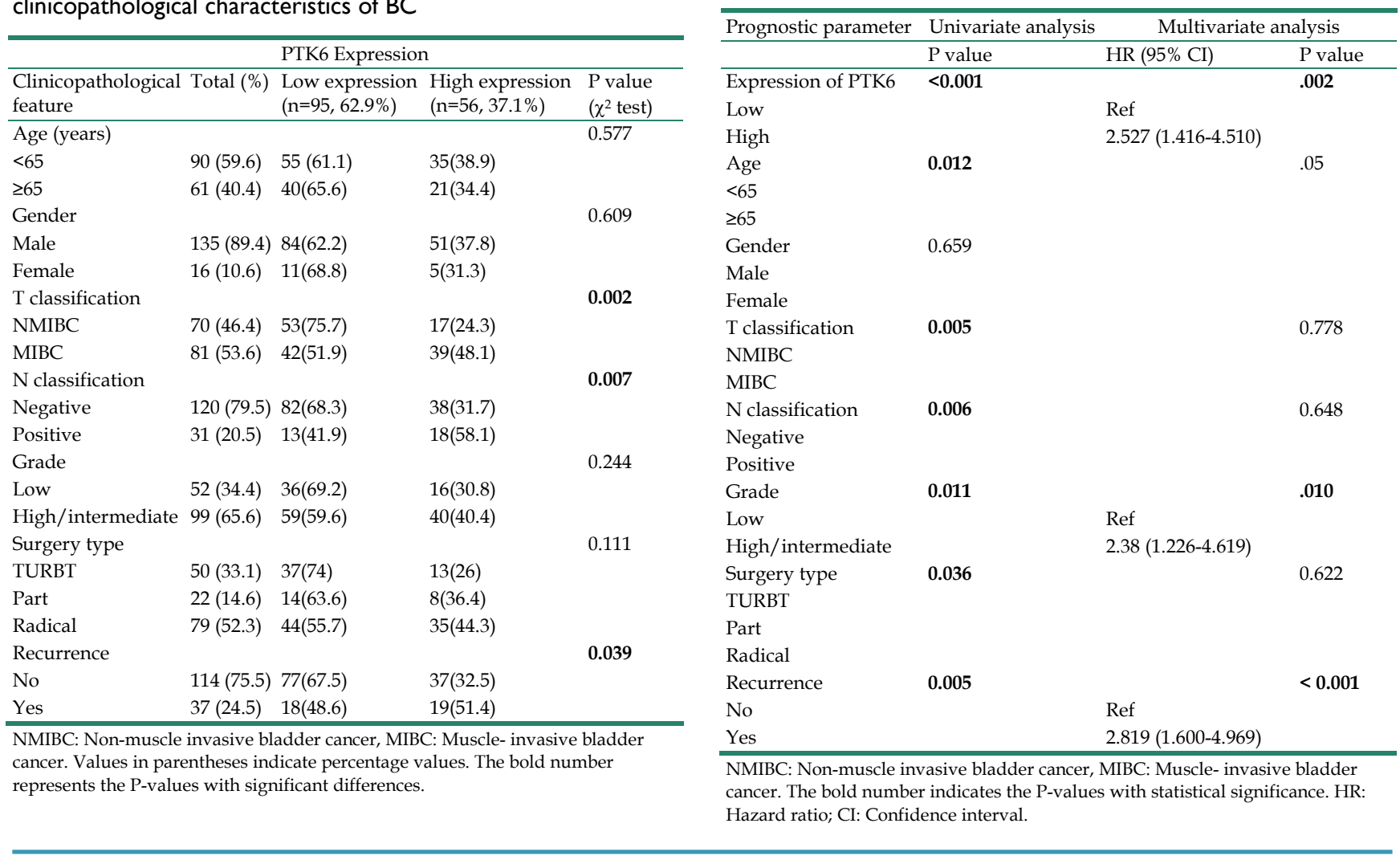

Table 3. Univariate and multivariate analyses of prognostic parameters for survival in patients with bladder cancer cancer. The bold number indicates the P-values with statistical significance. HR 
A

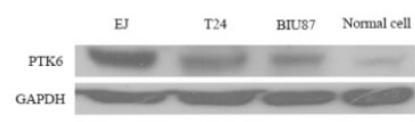

B

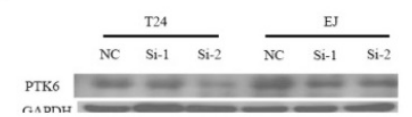

D
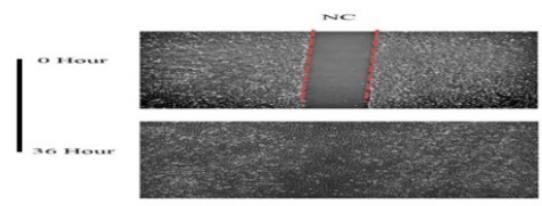

$\left.\right|_{\text {son }} ^{\infty}$

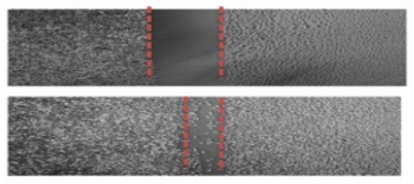

E
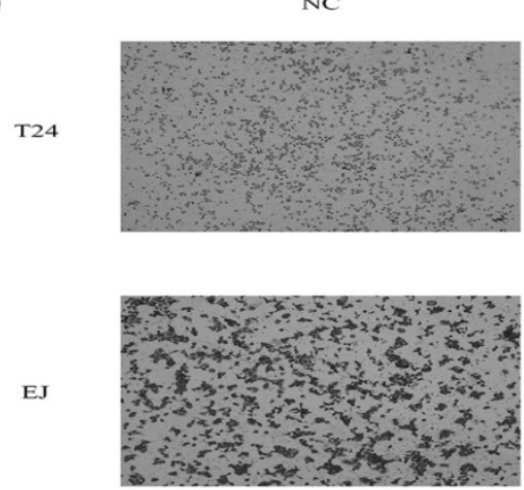

C
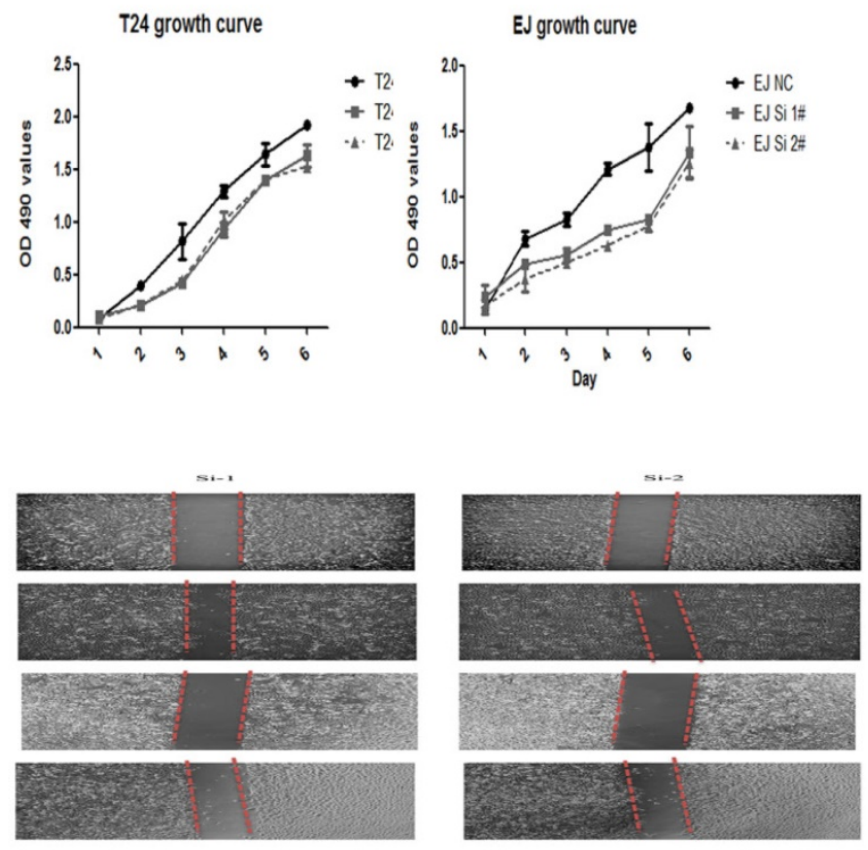

Si-1

Si-2
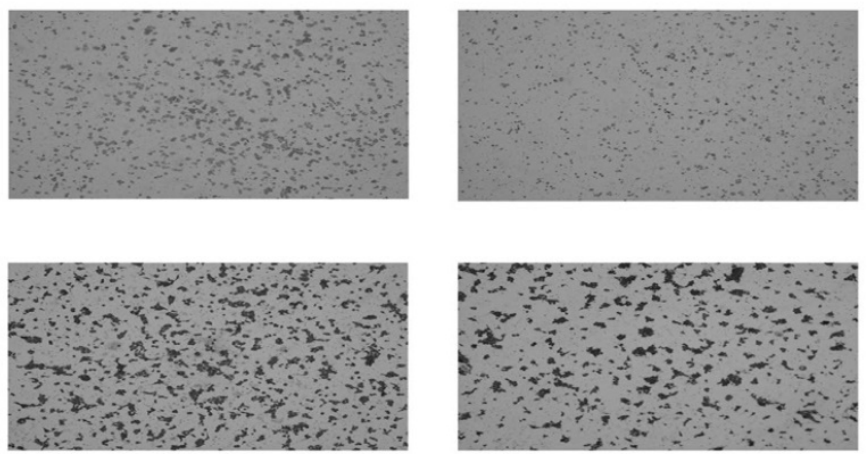

Figure 5: Knockdown of PTK6 inhibits the growth and migration of BC cell. (A) PTK6 expression was measured in three BC cell lines by western blot. (B) Western blot analysis of PTK6 expression in PTK6-depleting T24 and EJ cells. GADPH was used as the loading control. (C) The cell growth rates were determined by CCK-8 proliferation assays at various time points. (D-E) migration ability was measured by wound-healing and transwell chamber assay in T24 and EJ cells.

\section{Discussion}

Although PTK6 is involved in carcinogenesis and tumor progression in multiple studies [15, 22, 25], the relationship between PTK6 and BC is poorly understood. In the present study, we demonstrated that the expression of PTK6 was significantly elevated in BC tissues compared with corresponding adjacent non-cancerous tissues by RT-PCR and western blot. These findings are consistent with Oncomine analysis, which highlights the consistent trends in BC. According to the meta-analysis across 3 Oncomine datasets, we also revealed PTK6 was among one of the most upregulated genes in bladder cancer. Moreover, we extended our analysis to a non-overlapping cohort of 151 patients and found that PTK6 overexpression in tissue sections is strongly associated with the $\mathrm{T}$ classification, $\mathrm{N}$ classification and recurrence. Importantly, patients with PTK6 overexpression had significant shorter survival time than those with low expression. Cox proportional hazard regression analysis further identified PTK6 as an independent factor for poor prognosis. Taken together, these observations strongly indicated that PTK6 may be a cancer promoter for $\mathrm{BC}$ and may play a significant role in $\mathrm{BC}$ progression.

PTK6, the cytoplasmic non-receptor tyrosine kinase, plays a critical role in tumor initiation, progression and metastasis. Previous study disclosed that PTK6 localized at the plasma membrane 
promotes cell proliferation and migration [22]. However, the biological function of PTK6 in BC still remain elusive. According to predictive results obtained by bioinformatics, we detected that cell proliferation and metastasis-related genes were enriched in PTK6 overexpression group compared with PTK6 lower expression group. Function assays showed that BC cell growth and migration were significantly inhibited by knockdown of PTK6.

Proliferation and metastasis are a leading cause of cancer-related death, leading to a poor prognosis. Previous research has demonstrated that PTK6 inhibitor promotes breast cancer cell metastasis and apoptosis [22, 26, 27]. In this study, we discovered the cell growth and migration abilities of T24 and EJ cells were attenuated after knockdown of PTK6, which was supported by our clinical data and GSEA analysis. In summary, these data indicate that PTK6 can facilitate bladder cancer cell proliferation and metastasis. These findings were consistent with previous studies $[15,17$, 22].

In conclusion, computational analysis of high volume data was used to predict the oncogenic role of PTK6. Elevated PTK6 expression is associated with poor survival in BC patients, suggesting that PTK6 can be considered a prognostic or therapeutic biomarker in patients with BC. Therefore, we suggest that small molecule inhibitor of PTK6 can also be carried out in BC.

\section{Abbreviations}

BC: bladder cancer; PTK6: Protein tyrosine kinase 6; GSEA: gene set enrichment analysis; OS: Overall survival.

\section{Acknowledgments}

This work was supported by the National Natural Science Foundation of China (Grant No. 81072101).

\section{Ethics statement}

The use of tissues for this study has been approved by the Ethical Committee of Sun Yat-Sen University Cancer Center. At the time of initial diagnosis, all patients had provided written consent in the sense that their tumor samples could be used for the investigation's purpose.

\section{Competing Interests}

The authors have declared that no competing interest exists.

\section{References}

1. Siegel RL, Miller KD and Jemal A. Cancer Statistics, 2017. CA: a cancer journal for clinicians. 2017; 67(1):7-30.
2. Torre LA, Bray F, Siegel RL, Ferlay J, Lortet-Tieulent J and Jemal A. Global cancer statistics, 2012. CA: a cancer journal for clinicians. 2015; 65(2):87-108.

3. Ferlay J, Shin HR, Bray F, Forman D, Mathers C and Parkin DM. Estimates of worldwide burden of cancer in 2008: GLOBOCAN 2008. International journal of cancer. 2010; 127(12):2893-2917.

4. Sanli O, Dobruch J, Knowles MA, Burger M, Alemozaffar M, Nielsen ME and Lotan Y. Bladder cancer. Nature reviews Disease primers. 2017; 3:17022.

5. Xia L, Taylor BL and Guzzo TJ. Characteristics and Associated Factors of Postoperative Pulmonary Complications in Patients Undergoing Radical Cystectomy for Bladder Cancer: A National Surgical Quality Improvement Program Study. Clinical genitourinary cancer. 2017.

6. Kukreja JB and Shah JB. Advances in surgical management of muscle invasive bladder cancer. Indian journal of urology : IJU : journal of the Urological Society of India. 2017; 33(2):106-110.

7. Kang M, Jeong CW, Kwak C, Kim $\mathrm{HH}$ and $\mathrm{Ku}$ JH. Single, immediate postoperative instillation of chemotherapy in non-muscle invasive bladder cancer: a systematic review and network meta-analysis of randomized clinical trials using different drugs. Oncotarget. 2016; 7(29):45479-45488.

8. Chen L, Pan XW, Huang H, Gao Y, Yang QW, Wang LH, Cui XG and Xu DF. Epithelial-mesenchymal transition induced by GRO-alpha-CXCR2 promotes bladder cancer recurrence after intravesical chemotherapy. Oncotarget. 2017.

9. Ba M, Cui S, Wang B, Long H, Yan Z, Wang S, Wu Y and Gong Y. Bladder intracavitary hyperthermic perfusion chemotherapy for the prevention of recurrence of non-muscle invasive bladder cancer after transurethral resection. Oncology reports. 2017; 37(5):2761-2770.

10. Lu CS, Shieh GS, Wang CT, Su BH, Su YC, Chen YC, Su WC, Wu P, Yang WH, Shiau $\mathrm{AL}$ and $\mathrm{Wu} \mathrm{CL}$. Chemotherapeutics-induced Oct4 expression contributes to drug resistance and tumor recurrence in bladder cancer. Oncotarget. 2017; 8(19):30844-30858

11. Lee H, Kim M, Lee KH, Kang KN and Lee ST. Exon-intron structure of the human PTK6 gene demonstrates that PTK6 constitutes a distinct family of non-receptor tyrosine kinase. Molecules and cells. 1998; 8(4):401-407.

12. Mathur PS, Gierut JJ, Guzman G, Xie H, Xicola RM, Llor X, Chastkofsky MI, Perekatt $\mathrm{AO}$ and Tyner AL. Kinase-Dependent and -Independent Roles for PTK6 in Colon Cancer. Molecular cancer research : MCR. 2016; 14(6):563-573.

13. Mitchell PJ, Barker KT, Martindale JE, Kamalati T, Lowe PN, Page MJ, Gusterson BA and Crompton MR. Cloning and characterisation of cDNAs encoding a novel non-receptor tyrosine kinase, brk, expressed in human breast tumours. Oncogene. 1994; 9(8):2383-2390.

14. Easty DJ, Mitchell PJ, Patel K, Florenes VA, Spritz RA and Bennett DC. Loss of expression of receptor tyrosine kinase family genes PTK7 and SEK in metastatic melanoma. International journal of cancer. 1997; 71(6):1061-1065.

15. Chen X, Song B, Lin Y, Cao L, Feng S, Zhang L and Wang F. PTK6 promotes hepatocellular carcinoma cell proliferation and invasion. American journal of translational research. 2016; 8(10):4354-4361.

16. Kasprzycka M, Majewski M, Wang ZJ, Ptasznik A, Wysocka M, Zhang Q, Marzec M, Gimotty P, Crompton MR and Wasik MA. Expression and oncogenic role of Brk (PTK6/Sik) protein tyrosine kinase in lymphocytes. The American journal of pathology. 2006; 168(5):1631-1641.

17. Ono $\mathrm{H}$, Basson MD and Ito H. PTK6 promotes cancer migration and invasion in pancreatic cancer cells dependent on ERK signaling. PloS one. 2014; 9(5):e96060.

18. Harvey AJ and Crompton MR. Use of RNA interference to validate Brk as a novel therapeutic target in breast cancer: Brk promotes breast carcinoma cell proliferation. Oncogene. 2003; 22(32):5006-5010.

19. Chen YF, Ma G, Cao X, Huang ZL, Zeng MS and Wen ZS. Downregulated expression of PTK6 is correlated with poor survival in esophageal squamous cell carcinoma. Medical oncology. 2014; 31(12):317.

20. Ma S, Bao JY, Kwan PS, Chan YP, Tong CM, Fu L, Zhang N, Tong AH, Qin YR, Tsao SW, Chan KW, Lok S and Guan XY. Identification of PTK6, via RNA sequencing analysis, as a suppressor of esophageal squamous cell carcinoma. Gastroenterology. 2012; 143(3):675-686 e671-612.

21. Liu XK, Zhang XR, Zhong Q, Li MZ, Liu ZM, Lin ZR, Wu D and Zeng MS. Low expression of PTK6/Brk predicts poor prognosis in patients with laryngeal squamous cell carcinoma. Journal of translational medicine. 2013; 11:59.

22. Shin WS, Shim HJ, Lee YH, Pyo M, Park JS, Ahn SY and Lee ST. PTK6 Localized at the Plasma Membrane Promotes Cell Proliferation and MigratiOn Through Phosphorylation of Eps8. Journal of cellular biochemistry. 2017.

23. Sanchez-Carbayo M, Socci ND, Lozano J, Saint F and Cordon-Cardo C. Defining molecular profiles of poor outcome in patients with invasive bladder cancer using oligonucleotide microarrays. Journal of clinical oncology : official journal of the American Society of Clinical Oncology. 2006; 24(5):778-789.

24. Dyrskjot L, Kruhoffer M, Thykjaer T, Marcussen N, Jensen JL, Moller K and Orntoft TF. Gene expression in the urinary bladder: a common carcinoma in situ gene expression signature exists disregarding histopathological classification. Cancer research. 2004; 64(11):4040-4048

25. Zheng $\mathrm{Y}$ and Tyner AL. Context-specific protein tyrosine kinase 6 (PTK6) signalling in prostate cancer. European journal of clinical investigation. 2013; 43(4):397-404.

26. Ito K, Park SH, Nayak A, Byerly JH and Irie HY. PTK6 Inhibition Suppresses Metastases of Triple-Negative Breast Cancer via SNAIL-Dependent E-Cadherin Regulation. Cancer research. 2016; 76(15):4406-4417. 
27. Park SH, Ito K, Olcott $\mathrm{W}$, Katsyv I, Halstead-Nussloch $\mathrm{G}$ and Irie HY. PTK6 inhibition promotes apoptosis of Lapatinib-resistant Her2(+) breast cancer cells by inducing Bim. Breast cancer research : BCR. 2015; 17:86.

28. Zeng M, Yang Z, Hu X, Liu Y, Yang X, Ran H, Li Y, Li X and Yu Q. Grb7 gene amplification and protein expression by FISH and IHC in ovarian cancer. International journal of clinical and experimental pathology. 2015; 8(9):11296-11304. 